\title{
A contribution on manufacturing process development for composite material forming
}

\author{
Philippe MAYER ${ }^{1, a^{*}}$, Henri PERRIN ${ }^{1, b}$, Eric BECKER ${ }^{2, c}$, \\ Laurent LANGLOIS ${ }^{2, \mathrm{~d}}$, Régis BIGOT${ }^{2, \mathrm{e}}$ \\ ${ }^{1}$ PPE (Pôle de Plasturgie de l'Est), 18, Avenue Général Patton, 57500 Saint-Avold, FRANCE \\ ${ }^{2}$ ENSAM (Ecole Nationale Supérieure des Arts et Métiers), 4, Rue Augustin Fresnel, 57078 Metz, \\ FRANCE
}

a p.mayer@ppe.asso.fr , bh.perrin@ppe.asso.fr , ceric.becker@ensam.eu ,

${ }^{\mathrm{d}}$ laurent.langlois@ensam.eu, ${ }^{\mathrm{e}}$ regis.bigot@ensam.eu

Keywords: composite parts, shape forming, composite anisotropy, continuous fibers, transversal flow, squeezing.

\begin{abstract}
The author presents an approach to study an innovative manufacturing process developed to produce composite parts with new geometrical possibilities. The aim of this first study is to understand the motions of fibers during the forming of this kind of composite parts and to explore the feasibility of this forming process by an experimental analysis and FEM simulations with Forge 3C. The reliability of the simulation tool and its potential, never exploited in this area, will be evaluated.
\end{abstract}

\section{Introduction}

Car manufacturers are submitted to new regulations in order to reduce $\mathrm{CO} 2$ emissions. The objectives for 2020 have already been established by the European community. Several ways are identified to face these new challenges, such as the "downsizing" on the brand new motors, popular in the last years; or the "lightening" of the cars by reducing the structural parts weight. The latter requires the use of different materials to create lighter structures as efficient as the old ones.

Automotive industry creates partnerships with composite manufacturers to find new ways to manufacture tomorrow cars. In this context, the LCFC (Laboratoire de Conception, Fabrication et Commande) and the PPE (Pôle de Plasturgie de l'Est) decided to use their knowledge in Forging and composite manufacturing to create a new process for the automotive and aeronautic industries. The objectives are to create structural parts, by forming massive composite preform over the glass transition temperature.

Massive parts must be mechanically characterized and their integrity must be healthy with minimal voids contents and maximal fibers impregnation. These characterizations must be done in order to improve the contacts between the composite components and create a predictable composite structure. The simulations, were performed with the well-known forging software called Forge $\mathbb{C}$, with the aim of evaluating the capacity of the simulation tool in this new composite manufacturing process. 


\section{Process position in the composite forming industry}

The process presented in this article was developed in order to answer to the automotive industry demands concerning the manufacturing of lighter parts. To produce structural parts for the automotive industry, two "sine qua non" conditions are established: parts must be resistant to the structural constraints imposed and they should be produced at high speed.

In term of performance, the objective is to place the process near the CFRT (Continuous fiber reinforced thermoplastic), and the winding method. Both of them use continuous fibers at high fiber volume ratio with the purpose of creating highly resistant products.

In term of production rate, the objective is to place the process near the SMC (sheet molding compound) and the BMC (bulk molding compound) methods because they are currently used at high rate productions.

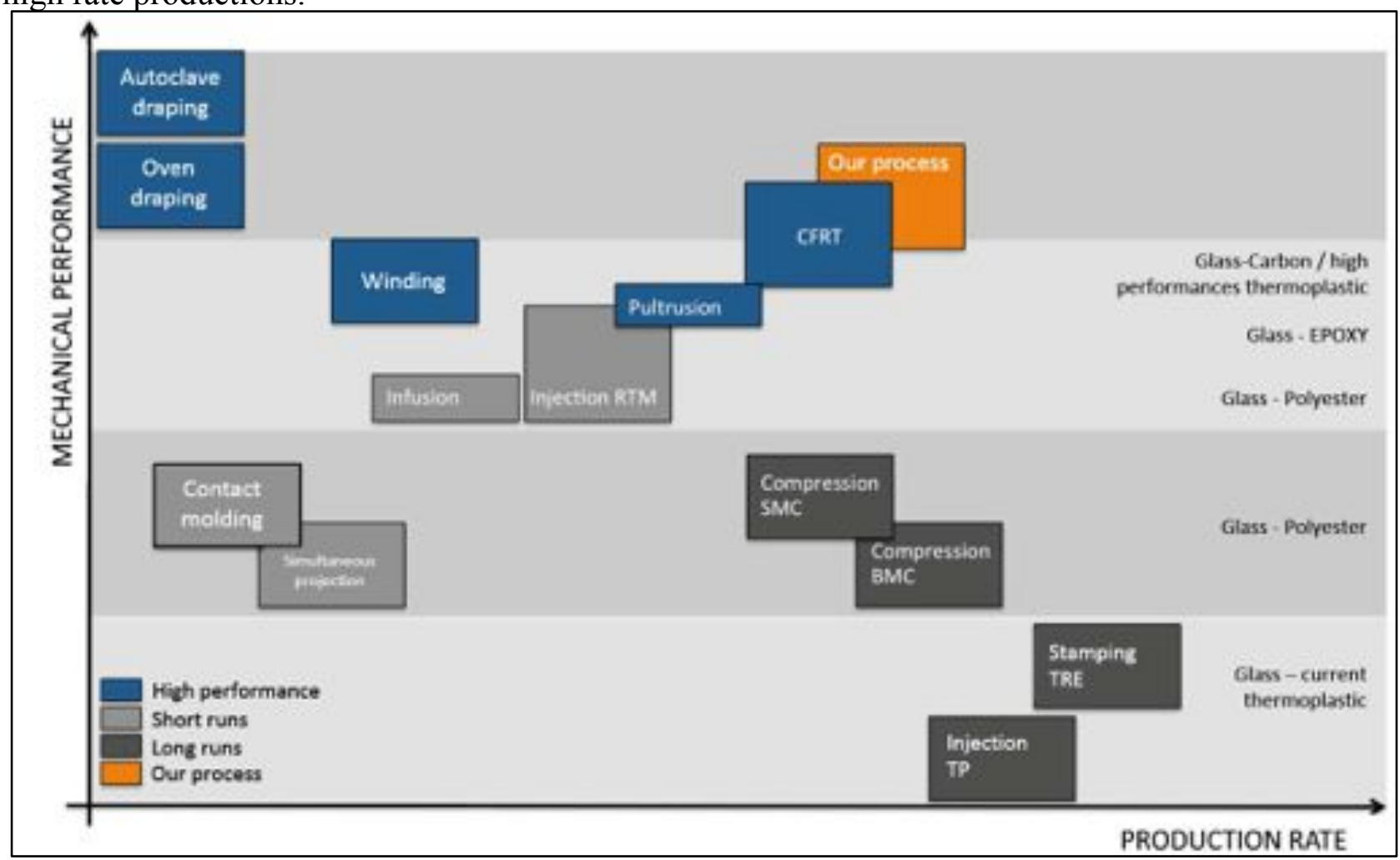

Figure 1 : Placement of the process in the composite manufacturing family

\section{Previous experimental and simulational investigations}

At the beginning of the project, the laboratory decided to study the forming of continuous fibers composite because of their high mechanical performances. In this first step of the study, it was decided to use pre-impregnated unidirectional composite. The material chosen allowed a constant fiber volume ratio with an easy use. The parts obtained during the experimental phase were compared to the bi-material simulation results of the forming. By this comparison it was also possible to analyze the capacity and reliability of the FEM simulation.

\section{a. Experimental investigations}

The basic idea of the manufacturing process is resumed in two steps:

- The first step is to create a simple preform, with continuous fibers.

- The second step is to form this preform, and to give the product special characteristics (whether morphological features or physical characteristics). 
This study concerns massive parts. A massive part is defined by the comparison between the thickness and the exterior radius of a cylinder (in the case of a simple winding) [1][2].

The manufacturing process in this research work is described as follow:

1) The fibers are impregnated outside before the forming. The pre-impregnated continuous carbon fibers with polypropylene from the preform presents a fibers volume ratio around $50 \%$.

2) Forming the preform.

3) At the end of the forming, the product contains about $50 \%$ of fibers in its volume.
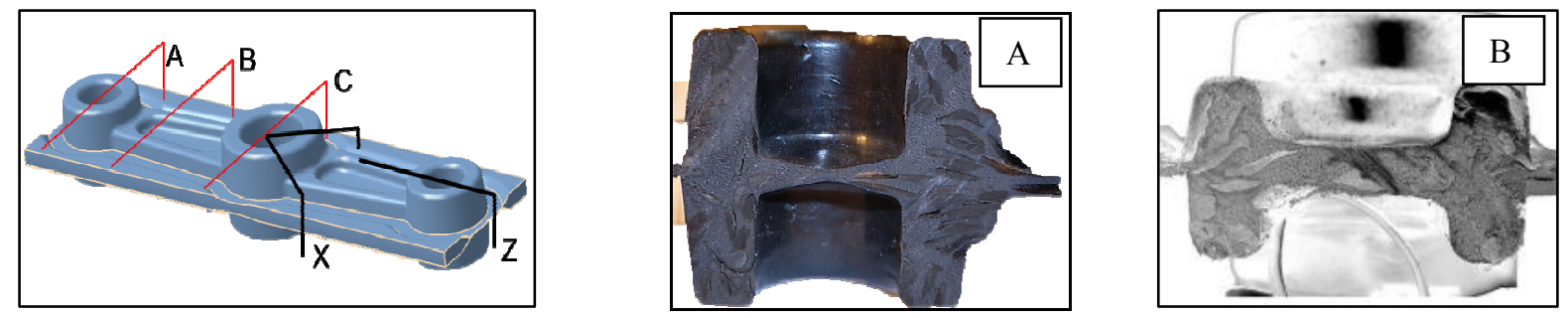

Figure 2 : Fibers displacements on two different areas $A$ and $B$

After forming, one of the test done was to evaluate the influence of the fibers start/finish position on fibers impregnation (see Figure 2). The part undergoes high constraints during forming and the fibers impregnation presents a real interaction with the hydrostatical pressure induced by the tools.

The simulations were developed to show this observation, and to present a significant dependence between the fibers impregnation and the pressure exercised by the melted polymer.

b. Simulational investigation

The simulations were performed with the software Forge3(C). A Bi-material simulation model was used with a Newtonian polymer behavior and a deformable part for the fibers. The experimental and simulation results were compared by analyzing across section of a part. (see Figure 3).
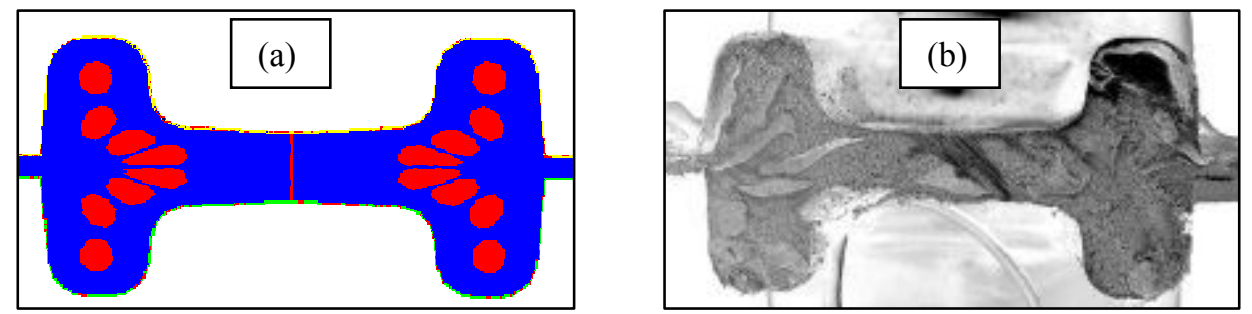

Figure 3 : Comparison of the displacements of the fibers between the (a) bi-material simulation and a (b) experimental results on the real-part.

The use of this simulation method gave some conclusive results:

$\checkmark$ The bi-material procedure presented correct displacements of the fibers in many cases.

$\checkmark$ The impregnation of the fibers became higher at the center of the part at higher values of pressure. 
The simulation results were in good agreement with the experimental observations. Two conclusions were identified: (1) the pressure has an important effect on the displacement of the fibers and their impregnation (very important for the mechanical limits of the part). This relation is due to the non-Newtonian viscosity of the melted polymer. The difference between the simulations and the real-parts can be explained by the behavior of the melted polymer which is inducing pressure fields during the processing. To increase the efficiency of the simulations, it is necessary to optimize the rheological behavior of the material in the simulation software, in order to cover all the shear rates cases. (2) The preparation of the simulation must be optimized (remeshing process) especially for this bi-material calculation method.

For the next stages of the study, commingled composites will be used because of their wide application possibilities. Unfortunately, these composites have some drawbacks during the high pressures forming, like some displacements of the melted polymer between the fibers [3].

\section{Discussion about current work}

The previous research work permitted to understand the behavior of composites during the manufacturing process. The composite transverse rheology at melted temperature is considered as an essential property of the process. In the literature, this physical behavior is defined by two terms : the transverse flow of CFRT (continuous fibers reinforced thermoplastics) [4] and the squeezing of CFRT [5]. In these studies, the authors precise a significant relation between the transverse viscosity and the fiber volume ratio. This relation is important in the case of thick structural parts. The pressures, and then the induced deformations during the forming, are clearly dependent on the intrinsic rheology of the composite.

The study was separated in two steps in order to understand the physical phenomenon:

- In the first step a numerical model is created, and computed in a simulation tool like Abaqus $(\mathbb{C}$ or Forge $\subset$. This model is a rheological model, considering in a first time the composite and his transverse viscosity with a Carreau-Yasuda [6] and Arrhenius coupled law [7]. The objective is to compare the void content in a part and the constraint distribution in the numerical model with a simple experiment. An important point is that the tool and the part will be heated at the same time in a laboratory environment to minimize the interaction between the devices and the composite. The model will be validated for a low cadence.

- In second step, the numerical model will be confronted to another case. The objective is to validate the heating of the composite outside a thermo-regulated mold, in order to validate a higher cadence.

The first step was started with a simple macro simulation in order to show the pressure during the forming. The melted polymer has a rheological behavior different than current materials used in Forge $($ C (Steel most of the time). A Carreau-Yasuda model, with a thermo-dependence with Arrhenius was implemented point to point in order to cover all the shear rates of the model. 


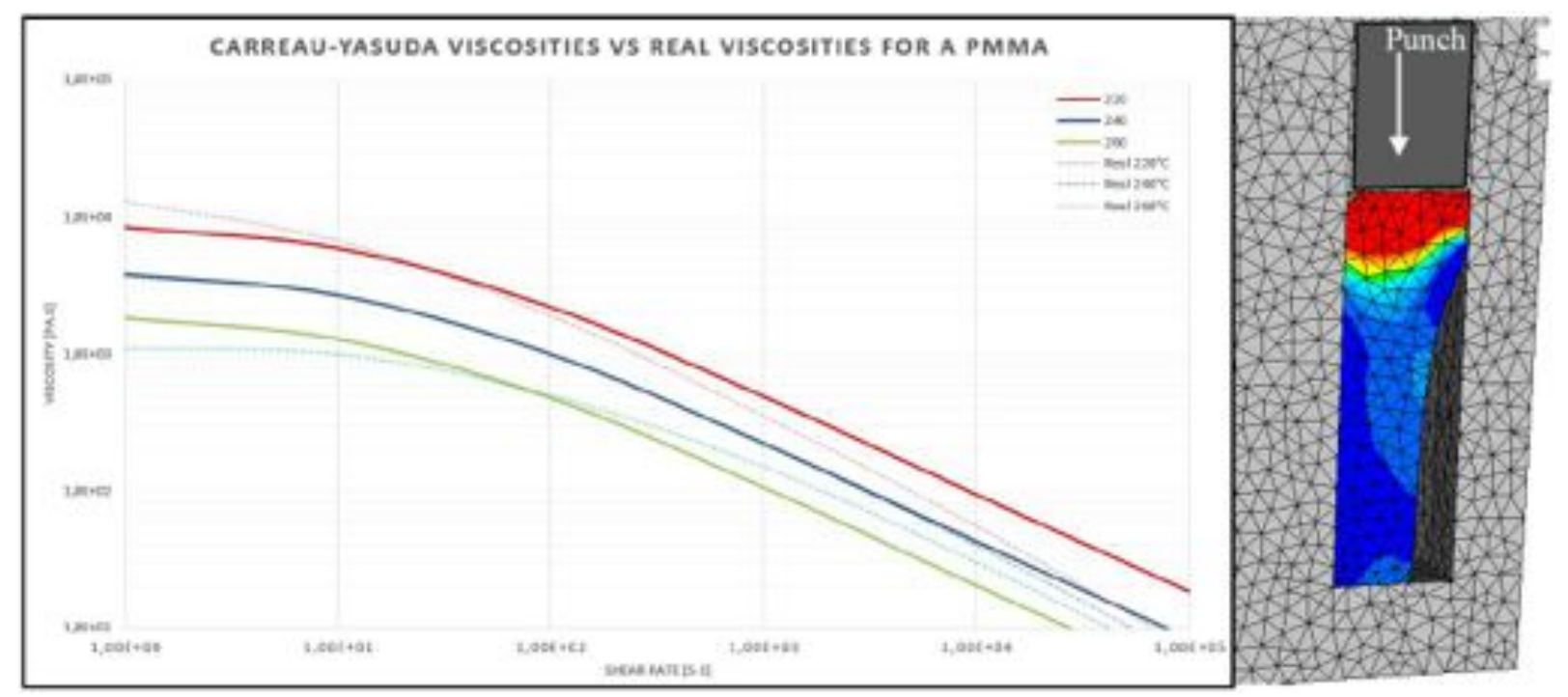

Figure 4 : (a) Carreau-Yasuda comparison for a PMMA \& Forge C simulation, reference temperature $240^{\circ} \mathrm{C}-(\mathrm{b})$ forming a polymer at melted temperature, red = maximum pressure, blue = minimum pressure

The simulations confirm that the pressure in the melted polymer part has a special distribution, and that the pressure field history of the part cannot be neglected to predict the distribution of the fibers in the final part.

This model was implemented in the software with success and the forming simulations of a simple thermoplastic cylinder was realized. The next step of the study was to implement a macro/micro model in Forge $\subset$ with transverse and longitudinal viscosities of the composite, and compare it to a forged part, done at a laboratory size. The fiber volume ratio and the void contents will be measured up to the normalization [8] compared to the hydrostatical pressure founded by the simulations. We defined how to input a coupling between the viscosity and the fiber volume ratio and implement it in a simulation tool by a simple algorithm. The initial part has different area, with different fiber ratio. These fibers ratios induce behavior law on a local area. Coupled to boundary conditions, through an increment of time, the simulation tool calculates the pressure field, and then the displacements of the fibers. The fibers volume ratio is recalculated and the coupling starts again.

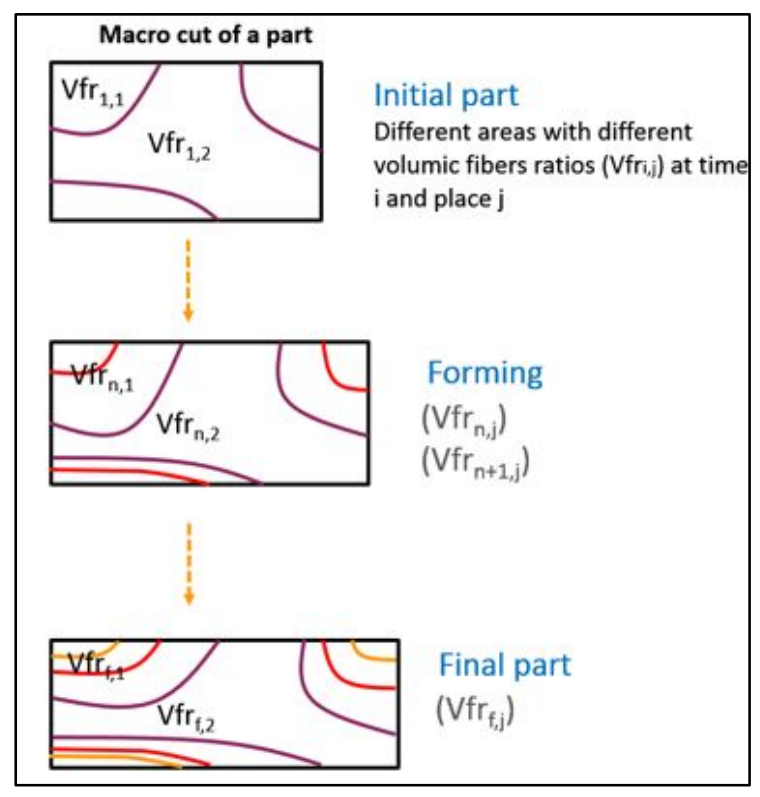

Figure 5 : Evolution of volumic fiber ratio 


\section{Conclusions \& Outlooks}

An initial step was realized to identify:

- The influence of the process operating parameters, like the viscosity and the temperature.

- A link between the fibers impregnation and the pressure field history induced by the forming.

The observation of the cut part shows that the fibers impregnation and their placement are dependent on the hydrostatic pressure induced by the tool, and by the processing parameters.

As outlooks, a model which is coupling a Carreau-Yasuda/Arrhenius behavior to a mechanical continuous element with the properties of carbon fibers will be confronted to a real part. This comparison will validate the link between the void content, the displacement of the fibers, the fiber volume ratio and the pressure field history of the part. The aim is to quantify the link observed between the displacement of the fibers and the pressure fields induced by the process, and characterize the potential of it with different materials, at different processing settings. In order to master all the forming operations, the cartography of the pressure field's history obtained in the simulations will be used.

\section{Bibliography}

[1] A. Pilato, "Caractérisation des structures composites bobinées épaisses, application a l'étude du comportement de réservoirs de stockage d'hydrogène," 2011.

[2] A. K. Roy and S. W. Tsai, "Design of Thick Composite Cylinders," vol. 110, no. August 1988, 2014.

[3] M. D. Wakeman, T. A. Cain, C. D. Rudd, R. Brooks, and A. C. Long, "Compression moulding of glass and polypropylene composites for optimised macro- and micromechanical properties - commingled glass and polypropylene," vol. 3538, no. 98, 1998.

[4] J. a. Barnes and F. N. Cogswell, "Transverse flow processes in continuous fibre-reinforced thermoplastic composites," Composites, vol. 20, no. 1, pp. 38-42, Jan. 1989.

[5] C. Coleman, "On the squeezing flow of a fibre-reinforced fluid," J. Nonnewton. Fluid Mech., vol. 37, pp. 379-385, 1990.

[6] S. . Shuler and S. . Advani, "Transverse squeeze flow of concentrated aligned fibers in viscous fluids," J. Nonnewton. Fluid Mech., vol. 65, no. 1, pp. 47-74, Jul. 1996.

[7] C. M. Djebali, "Rhéologie des polymères fondus à hauts taux de cisaillement; application à la microinjection," 2012.

[8] D. D. Adams, "Composite Structure Engineering Safety Awareness Course - Composite Materials Test Methods," vol. 84112, no. 801, 2010. 